\title{
Physiological coreceptor use by dual-tropic HIV-1: one plus one equals one
}

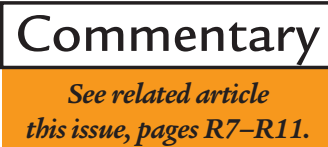

\author{
Barrett J. Rollins \\ Department of Adult Oncology, Dana-Farber Cancer Institute; Department of Medicine, Brigham \& Women's Hospital and \\ Harvard Medical School, Boston, Massachusetts 02115, USA \\ Address correspondence to: Barrett J. Rollins, Department of Adult Oncology, M430, Dana-Farber Cancer Institute, 44 Binney Street, \\ Boston, Massachusetts 02115, USA. Phone: (617) 632-3896; Fax: (617) 632-5998; E-mail: barrett_rollins@dfci.harvard.edu.
}

The AIDS epidemic has provided virologists and immunologists with any number of puzzles and mysteries. Until recently, one of the less comprehensible behaviors of HIV-1 was its species specificity. In spite of the fact that CD4 is clearly its receptor, the virus will not infect murine cells engineered to express human CD4. An allied, and no less opaque, problem is that not all CD4expressing human cells are infectible by all strains of HIV-1. Some strains, particularly those isolated early in infection, will only infect macrophages or primary activated T cells (called M-tropic strains), while those isolated from patients with AIDS will readily infect both primary activated $\mathrm{T}$ cells and $\mathrm{T}$ lymphocyte cell lines (called T-tropic, or more properly, T-cell line-tropic [TCLtropic] strains). Some of these later isolates are exclusively TCL-tropic, but most have kept their M-tropism and are denoted as dual-tropic viruses $(1,2)$.

HIV-1 infection begins with fusion of the viral envelope to the plasma membrane of the target cell. A variety of

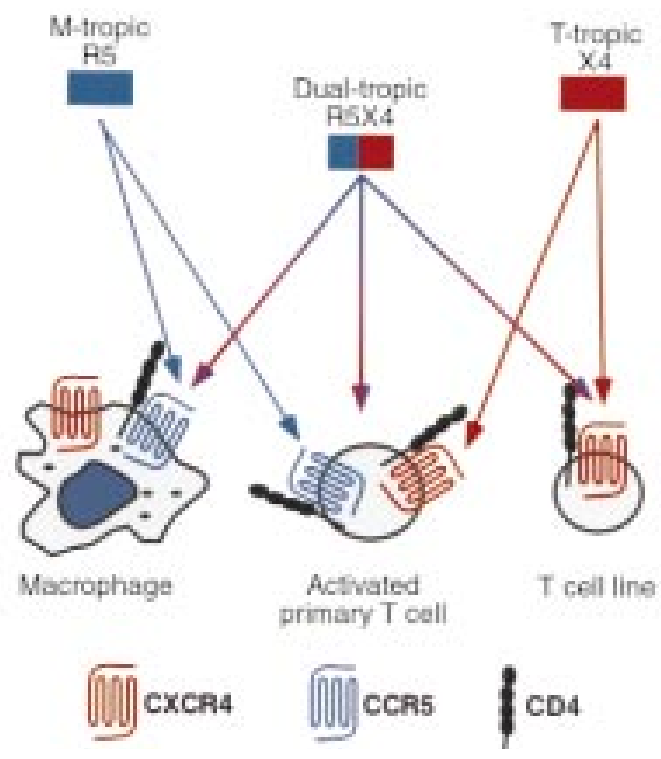

ingenious assays that specifically examine this stage of the viral life cycle provide a readout of membrane fusion without depending on any subsequent events, such as viral replication (3). Using these assays, it became clear that uninfectible cells could be rendered infectible by physically fusing them with susceptible cells. As the only function measured in this assay is viral fusion, that observation implied the existence of a second receptor, or coreceptor, working in concert with CD4 to permit fusion and infection. But the molecular nature of the coreceptor was another one of those enduring HIV mysteries.

Relief came abruptly in 1996 with Ed Berger's landmark discovery that the coreceptor used by TCL-tropic strains is the chemokine receptor CXCR4 (4). With that paradigm established, several groups promptly demonstrated that the primary receptor used by M-tropic strains is another chemokine receptor, CCR5 (5-8). This explained the observation that 3 chemokines known to be ligands for CCR5 - MIP- $1 \alpha$, MIP- $1 \beta$, and RANTES - could prevent infection by $\mathrm{M}$-tropic, but not TCL-tropic, viruses (9). Conversely, the ligand for CXCR4, SDF-1 $\alpha$, prevents infection by TCLtropic, but not M-tropic, viruses $(10,11)$.

A model can now be constructed that unites strain tropism with the course of clinical disease (Figure 1). The transmissible strains of HIV-1 are almost exclusively $M$ tropic, and these are the strains isolated from patients during latency. These viruses are called R5 because they use

Figure 1

HIV-1 cellular tropism and chemokine coreceptors. See text for details.
CCR5, but not CXCR4, and will not induce syncytia formation in T-cell lines. As disease progression occurs, strains acquire the ability to use CXCR4 and become either dual-tropic (R5X4) or exclusively TCL-tropic (X4). These viruses concurrently gain the ability to induce syncytia, although the relationship between this property and HIV pathogenesis is unclear.

Two observations have complicated this satisfyingly simple picture. The first is that the family of chemokine receptors is large, and many additional members have coreceptor activity in fusion assays in vitro (12). This raises the question of which coreceptors a virus actually uses in vivo. The second problem is the very existence of dualtropic viruses, and again raises the question of which coreceptors are pathophysiologically relevant.

In this issue of the JCI, Glushakova et al. (13) provide some answers to the latter question by examining coreceptor usage by dual-tropic viruses. They employ 2 technical approaches that lend weight to their assertion that despite dual tropism, these viruses use only 1 coreceptor in vivo. First, they infect intact fragments of human lymphoid tissue floating on collagen rafts. Because infection of cells in tissue culture requires activation, and because activation can modulate coreceptor expression, infection of intact lymphoid tissue more closely resembles infection in vivo and provides a more faithful model of coreceptor expression.

Their second technical trick is to use viruses that differ only in their cellular recognition sites, i.e., the viral envelope (14). The viral envelope protein is a 160$\mathrm{kDa}$ precursor that is cleaved into gp120 and gp41 subunits that, in turn, form noncovalently associated trimers in the membrane. A great deal of epitope analysis and a solved structural analysis indicate that gp120 binding to CD4 on a target cell produces a conformational 
change that enhances gp120 binding to its appropriate chemokine receptor $(15$, 16). There is also evidence that CD4 and the chemokine receptor preexist in a complex on the cell surface (17). Binding to the chemokine receptor then opens up gp120 to reveal gp41, which is the protein engine that drives membrane fusion.

At first glance, if one wanted to address the question of receptor usage by dual-tropic viruses, one would collect a handful of dual-tropic isolates and assay their specificities in cell lines and then in lymphoid rafts. The problem is that there is now substantial evidence that target cell specificity is not solely dictated by coreceptor usage or, if it is, it can be modified by cellular context or relative receptor number. For example, R4 viruses tend not to infect macrophages despite their expression of CXCR4. Some of these other determinants may differ depending on viral genetics, so that there is no guarantee that independently isolated dual-tropic viruses can be strictly compared.

Glushakova et al. get around this problem by using viruses that differ only in the region of gp120 that confers coreceptor specificity. Beginning with 89.6, a well-characterized dual-tropic virus, they created 2 additional viruses in which they replaced variable regions 3 , 4, and 5 of gp120 (V3-V5) with V3-V5 of the M-tropic strains SF162 or JR-FL. All 3 viruses were still dual-tropic in vitro, as defined by their ability to use CCR5 and CXCR4 to induce cell fusion. However, the authors then employed agents that block specific coreceptor usage to show that 89.6 exclusively uses CXCR4 in human lymphoid rafts, whereas the 89.6/SF162 chimera primarily used CCR5, and the 89.6/JR-FL chimera used both receptors. Furthermore, cytopathicity of each strain, as defined by $\mathrm{CD}^{+} \mathrm{T}$-cell depletion, correlated positively with its use of CXCR4 and negatively with its use of CCR5.

What does this tell us? The main message is that coreceptor use in vivo may not be predicted accurately by patterns of coreceptor use in vitro. This statement has to be draped with caveats. First, lymphoid rafts are not really the same as in vivo infection in humans. Second, chimeras were used to permit genetically clean comparisons, but that means that 2 of the 3 viruses examined do not exist in nature. And third, even though the chimeras both have an Mtropic V3 (the major determinant of coreceptor usage; ref. 14), they recognize CXCR4 in vitro just as well as 89.6, suggesting that the coreceptor interactions in the chimeras may not be physiological. Still, the point made with these 3 viruses echoes the observation that viruses capable of using several different chemokine receptors in vitro are nonetheless committed to using CCR5 exclusively when infecting PBMCs (18).

There is a striking analogy between the profusion of functional coreceptors defined by in vitro assays and the socalled promiscuity problem of the chemokine system (19). There are some 50 human chemokines and 18 defined receptors, with only a few unassigned orphans remaining. Ligand binding assays in vitro show, not unexpectedly, that many chemokines bind to more than 1 receptor, and many receptors bind more than 1 chemokine, all with high affinity. Because of this ligand/receptor promiscuity, many investigators have despaired of the possibility that there might be any specificity in the chemokine system. Targeting 1 chemokine or receptor with a drug was thought to be pointless, as the redundant ligand or receptor would step in to fill its physiologic shoes.

However, every chemokine ligand or receptor knockout mouse created so far has a unique phenotype. There is, as it turns out, tremendous inherent specificity in this system, and it appears to be based, in part, on when and where the chemokines and their receptors are expressed. In the same way, despite an ability to utilize many chemokine receptors in vitro, HIV strains examined in more physiological systems tend to restrict their usage. In the case of blood cells that express several receptors simultaneously, the basis for this selectivity is unclear. It may have to do with preferential association with CD4 or with postfusion events that are cell type-specific. In other cases, expression of certain coreceptors may be limited to specific anatomic sites, making them physiologically relevant for HIV infection at those loci.

The lesson, of course, is obvious and trite. There is no substitute for in vivo analysis. What the field desperately needs is a reliable and faithful in vivo model that would permit rigorous test- ing of the roles of specific coreceptors in HIV infection, thereby identifying drug targets with confidence. Until then, one must view in vitro data with caution, and realize that the use of 2 receptors in vitro may add up to the use of only 1 receptor in vivo.

1. Doranz, B.J., et al. 1996. A dual-tropic primary HIV-1 isolate that uses fusin and the betachemokine receptors CKR-5, CKR-3, and CKR-2b as fusion cofactors. Cell. 85:1149-1158.

2. Bjorndal, A., et al. 1997. Coreceptor usage of primary human immunodeficiency virus type 1 isolates varies according to biological phenotype. J. Virol. 71:7478-7487.

3. Broder, C.C., and Berger, E.A. 1995. Fusogenic selectivity of the envelope glycoprotein is a major determinant of human immunodeficiency virus type 1 tropism for CD4+ T-cell lines vs. primary macrophages. Proc. Natl. Acad. Sci. USA. 92:9004-9008.

4. Feng, Y., Broder, C.C., Kennedy, P.E., and Berger, E.A. 1996. HIV-1 entry cofactor: functional cDNA cloning of a seven-transmembrane, $G$ proteincoupled receptor. Science. 272:872-877.

5. Choe, H., et al. 1996. The beta-chemokine receptors CCR3 and CCR5 facilitate infection by primary HIV-1 isolates. Cell. 85:1135-1148.

6. Dragic, T., et al. 1996. HIV-1 entry into CD4+ cells is mediated by the chemokine receptor CC CKR-5. Nature. 381:667-673.

7. Alkhatib, G., et al. 1955. CC CKR5: a RANTES, MIP-1alpha, MIP-1beta receptor as a fusion cofactor for macrophage-tropic HIV-1. Science. 272:1955-1958.

8. Deng, H., et al. 1996. Identification of a major coreceptor for primary isolates of HIV-1. Nature. 381:661-666.

9. Cocchi, F., et al. 1995. Identification of RANTES, MIP- $1 \alpha$, and MIP- $1 \beta$ as the major HIV-suppressive factors produced by CD8 $+\mathrm{T}$ cells. Science. 270:1811-1815.

10. Bleul, C.C., et al. 1996. The lymphocyte chemoattractant SDF-1 is a ligand for LESTR/fusin and blocks HIV-1 entry. Nature. 382:829-833.

11. Oberlin, E., et al. 1996. The CXC chemokine SDF1 is the ligand for LESTR/fusin and prevents infection by T-cell-line-adapted HIV-1. Nature. 382:833-835.

12. Berger, E.A., Murphy, P.M., and Farber, J.M. 1999 Chemokine receptors as HIV-1 coreceptors: roles in viral entry, tropism, and disease. Annu. Rev. Immunol. 17:657-700.

13. Glushakova, S. et al. 1999. Preferential coreceptor utilization and cytopathicity by dual-tropic HIV1 in human lymphoid tissue ex vivo. J. Clin. Invest. 104:R7-R11.

14. Wyatt, R., and Sodroski, J. 1998. The HIV-1 envelope glycoproteins: fusogens, antigens, and immunogens. Science. 280:1884-1888.

15. Wyatt, R., et al. 1998. The antigenic structure of the HIV gp120 envelope glycoprotein. Nature. 393:705-711.

16. Kwong, P.D., et al. 1998. Structure of an HIV gp120 envelope glycoprotein in complex with the CD4 receptor and a neutralizing human antibody. Nature. 393:648-659.

17. Xiao, X., et al. 1999. Constitutive cell surface association between CD4 and CCR5. Proc. Natl. Acad. Sci. USA. 96:7496-7501.

18. Zhang, Y.J., and Moore, J.P. 1999. Will multiple coreceptors need to be targeted by inhibitors of human immunodeficiency virus type 1 entry? J. Virol. 73:3443-3448.

19. Rollins, B.J. 1997. Chemokines. Blood. 90:909-928. 